\title{
INTEGRALITY OF THE AVERAGED JONES POLYNOMIAL OF ALGEBRAICALLY SPLIT LINKS
}

\author{
HANS U. BODEN
}

This note arose out of an attempt to prove a conjecture of Lin and Wang concerning the the integrality of the coefficients of the Taylor series expansion at $t=1$ of the averaged Jones polynomial of algebraically split links. This question comes up in the study of Ohtsuki's invariants, $\lambda_{n}(M) \in \mathbb{Q}$, defined for $M$ an integral homology 3-sphere [, [W].

Suppose that $L$ is an oriented link in $S^{3}$ with $\mu$ components. Write the averaged Jones polynomial of $L$ as a Taylor series at $t=1$, i.e.

$$
\Phi(L ; t)=\sum_{i=0}^{\infty} a_{i}(t-1)^{i},
$$

and set $\phi_{n}(L)=(-2)^{\mu} a_{n+\mu}(L)$.

Conjecture 4.1 of [LW] states that for $L$ an algebraically split link (ASL),

$$
n ! \phi_{n}(L) \in 6 \mathbb{Z} .
$$

This conjecture is verified for $n=1,2$ in $\mathbb{L W}$, and we consider the case $n \geq 3$ here. We first establish that $a_{n}(L) \in \mathbb{Z}$ whenever $L$ is a geometrically split link (GSL), implying that $\phi_{n}(L) \in 2^{\mu} \mathbb{Z}$, which is a priori stronger than the conjecture in this case. Nevertheless, Conjecture 4.1 is not true for ASLs. The problem is the presence of additional factors of 2 in the denominator of $\phi_{n}(L)$.

The goal of this paper is to present two results, Proposition 1 for GSLs and Proposition 2 for ASL, both giving integrality results for the coefficients $a_{n}(L)$ which are sharp, as shown by examples (I) and (II).

For the definition of $\Phi(L ; t)$, see pp.10-11 of [LW]. It is roughly a sum of (normalized) Jones polynomials, summed over all sublinks of $L$, and it satisfies: 
(i) If $L$ is trivial or empty, then $\Phi(L ; t)=1$.

(ii) If $L=L_{1} \cup L_{2}$ is a geometric splitting, then $\Phi\left(L_{1} \cup L_{2} ; t\right)=\Phi\left(L_{1} ; t\right) \cdot \Phi\left(L_{2} ; t\right)$.

This Jones polynomial satisfies a skein relation slightly different from the usual one and is normalized by dividing by $\left(t^{1 / 2}+t^{-1 / 2}\right)^{\mu}$ (see p.11, [LW]). For example, if $L$ is Brunnian (i.e. all proper sublinks are trivial) and $J(L ; t)$ is the usual Jones polynomial (i.e. the one tabulated in C. Adams' book [A]), then

$$
\Phi(L ; t)=(-1)^{\mu}\left(1-\frac{J(L ; 1 / t)}{\left(t^{1 / 2}+t^{-1 / 2}\right)^{\mu}}\right) .
$$

This and property (ii) is all we need to know to settle the conjecture.

Define

$$
a_{n}(L)=\left.\frac{1}{n !} \frac{d^{n} \Phi(L ; t)}{d t^{n}}\right|_{t=1} .
$$

We claim that $a_{n}(L) \in \mathbb{Z}$ for $L$ a GSL. For knots $K$, it is evident that $\Phi(K ; t)$ is a Laurent polynomial, and property (ii) implies the same for $L$ a GSL, i.e. $\Phi(L ; t) \in$ $\mathbb{Z}[1 / t, t]$. Obviously the nth derivative of $t^{m}$ at $t=1$ is simply $m(m-1) \cdots(m-n+1)$, which is divisible by $n$ !, since any product of $n$ successive integers is divisible by $n$ ! (p. $63[\mathrm{HW}]$ ). It now follows by linearity that $\left.\frac{d^{n} \Phi(L ; t)}{d t^{n}}\right|_{t=1}$ is divisible by $n$ !, hence $a_{n}(L) \in \mathbb{Z}$. This implies Conjecture 4.1 for GSLs, but in fact, more is true.

For any ASL $L$, Theorem 4.1 and Lemma 4.2 of [LW] show that

$$
\Phi(L ; t)=\sum_{i=\mu+1}^{\infty} a_{i}(K)(t-1)^{i},
$$

(i.e. $a_{i}(L)=0$ for $i \leq \mu$ ), and that both $a_{\mu+1}(L)$ and $2 a_{\mu+2}(L) \in 3 \mathbb{Z}$. But if $\mu=1$ and $L=K$ is a knot, then $a_{n}(K) \in \mathbb{Z}$, hence $a_{3}(K) \in 3 \mathbb{Z}$.

Proposition 1. Suppose $L=K_{1} \cup \cdots \cup K_{\mu}$ is a GSL. Then

$$
\Phi(L ; t)=\sum_{i=2 \mu}^{\infty} a_{i}(L)(t-1)^{i},
$$

where

$$
\begin{array}{ll}
a_{i}(L) \in 3^{\mu} \mathbb{Z} & \text { for } 2 \mu \leq i \leq 3 \mu, \text { and } \\
a_{i}(L) \in 3^{4 \mu-i} \mathbb{Z} & \text { for } 3 \mu<i \leq 4 \mu, \\
a_{i}(L) \in \mathbb{Z} & \text { for } 4 \mu<i .
\end{array}
$$


Proof. By property (ii), we have

$$
\Phi(L ; t)=\Phi\left(K_{1} ; t\right) \cdots \Phi\left(K_{\mu} ; t\right)
$$

Multiplication of the series expansions of $\Phi\left(K_{j} ; t\right)=\sum_{i=0}^{\infty} a_{i}\left(K_{j}\right)(t-1)^{i}$ gives the formula

$$
a_{i}(L)=\sum_{\sigma_{1}+\cdots+\sigma_{\mu}=i} a_{\sigma_{1}}\left(K_{1}\right) \cdots a_{\sigma_{\mu}}\left(K_{\mu}\right) .
$$

The proposition now follows from the fact that $a_{0}\left(K_{j}\right)=0=a_{1}\left(K_{j}\right)$, and that $a_{2}\left(K_{j}\right)$ and $a_{3}\left(K_{j}\right)$ are multiples of 3 for $j=1, \ldots, \mu$.

Examples. (I) If $K$ is the left-hand trefoil, then

$$
\begin{aligned}
\Phi(K ; t) & =-t^{4}+t^{3}+t-1 \\
& =-3(t-1)^{2}-3(t-1)^{3}-1(t-1)^{4} .
\end{aligned}
$$

Taking $L$ to be the GSL $K \cup \cdots \cup K$ shows that Proposition 1 is sharp.

(II) Let $L$ be the Whitehead link. Consulting link tables凹, we obtain

$$
\begin{aligned}
\Phi(L ; t) & =\frac{-t^{7 / 2}+2 t^{5 / 2}-t^{3 / 2}+2 t^{1 / 2}-t^{-1 / 2}+t^{-3 / 2}}{t^{1 / 2}+t^{-1 / 2}}-1 \\
& =-t^{3}+3 t^{2}-4 t+5+t^{-1}-8(t+1)^{-1} . \\
& =\frac{-3(t-1)^{3}}{2}+\sum_{n=4}^{\infty} \frac{(-1)^{n}\left(2^{n-2}-1\right)(t-1)^{n}}{2^{n-2}} .
\end{aligned}
$$

In particular, $a_{5}(L)=-7 / 8$ and so $3 ! \phi_{3}(L)=21$, which provides a counterexample to Conjecture 4.1. Notice moreover that $\phi_{7}(L)=\frac{127}{32}$, thus $n ! \phi_{n}(L)$ need not be an integer.

Proposition 2. If $L$ is an $A S L$ with $\mu$ components, then

$$
2^{n-2} a_{n}(L) \in \mathbb{Z}
$$

\footnotetext{
${ }^{1} L=5_{1}^{2}$ in the standard notation [A]. Note that $J(L ; t)$ depends on a choice of orientation.
} 
Proof. For $L$ a GSL, this is a consequence of Proposition 1, while for $L$ an ASL, it follows by induction on $n, \mu$, and the double unlinking number, which is the number of double crossings needed to change $L$ to a GSL, as we now explain.

Consider two crossings, one positive and the other negative, between distinct components of $L$. Write $L=L_{+-}$, and notice that $L_{-+}$is an ASL with $\mu$ components, and that $L_{0+}$ and $L_{+0}$ are also ASL links, but with $\mu-1$ components. Using the skein relation twice and subtracting, we obtain the double crossing change formula (cf. Lemma 4.1, 때])

$$
(t+1)\left(\Phi\left(L_{+-} ; t\right)-\Phi\left(L_{-+} ; t\right)\right)=\left(t^{2}-t\right)\left(\Phi\left(L_{0+} ; t\right)-\Phi\left(L_{+0} ; t\right)\right) .
$$

Equating coefficients of the power series in equation (11) gives

$$
\begin{aligned}
a_{n}\left(L_{+-}\right) & =a_{n}\left(L_{-+}\right)+\frac{a_{n-1}\left(L_{-+}\right)-a_{n-1}\left(L_{+-}\right)}{2} \\
& +\quad \frac{a_{n-1}\left(L_{0+}\right)-a_{n-1}\left(L_{+0}\right)}{2}+\frac{a_{n-2}\left(L_{0+}\right)-a_{n-2}\left(L_{+0}\right)}{2} .
\end{aligned}
$$

The proposition now follows from this formula by induction, since we can assume that it has already been established for $L_{-+}, L_{0+}$, and $L_{+0}$, and that $2^{n-3} a_{n-1}\left(L_{+-}\right) \in \mathbb{Z}$. Note that the previous example indicates that this result is sharp.

\section{REFERENCES}

[A] C. Adams, The Knot Book, (1994) W. H. Freeman and Co., New York.

[HW] G. H. Hardy and E. M. Wright, The Theory of Numbers, (1968) fourth edition, Oxford University Press, London.

[LW] X.-S. Lin and Z. Wang, On Ohtsuki's Invariants of integral homology 3-spheres, I, (1995) preprint.

[O] T. Ohtsuki, A polynomial invariant of integral homology 3-spheres, Math. Proc. Camb. Phil. Soc., (1995), 117.

Department of Mathematics and Statistics, McMaster University, Hamilton, OnTARIO L8S 4K1 CANADA

E-mail address: boden@icarus.mcmaster.ca 